\title{
Osteomyelitis of the foot: non-surgical management, SPECT/CT scanning and minimising the duration of antibiotic use
}

\author{
William J. Jeffcoate ${ }^{1}$
}

Received: 17 August 2017 / Accepted: 30 August 2017 /Published online: 21 September 2017

(C) Springer-Verlag GmbH Germany 2017

\begin{abstract}
In a paper in this issue of Diabetologia (DOI: https://doi.org/10.1007/s00125-017-4417-x), Vouillarmet and colleagues have explored the use of single-photon emission computed tomography (SPECT)/computed tomography $(\mathrm{CT})$ to define remission during non-surgical management of osteomyelitis of the foot. Their experience in a non-controlled observational study of 45 individuals was that a negative white blood cell-SPECT/CT scan is a reliable marker of remission, while a positive scan at the end of antibiotic treatment may be relatively useful in the prediction of future short- to medium-term relapse. These findings and conclusions are discussed in the light of current uncertainties relating to the diagnosis of bone infection and the lack of any tested measure that can be used to indicate either its presence or its persistence. In this respect, it is concluded that the value of this approach, and in which population, remains to be clearly established.
\end{abstract}

Keywords Diabetic foot $\cdot$ Foot ulcer · Infection · MRI · Osteomyelitis $\cdot$ SPECT/CT

\author{
Abbreviations \\ CT Computed tomography \\ IWGDF International Working Group on the Diabetic Foot \\ NPV Negative predictive value \\ PPV Positive predictive value
}

William J. Jeffcoate

william.jeffcoate@gmail.com

1 Foot Ulcer Trials Unit, Department of Diabetes and Endocrinology, Nottingham University Hospitals Trust, City Hospital Campus, Nottingham NG5 1PB, UK
SPECT Single-photon emission computed tomography WBC White blood cell

\section{Introduction}

In this issue, Vouillarmet and colleagues [1] report the use of hybrid white blood cell (WBC)-single-photon emission computed tomography (SPECT)/x-ray computed tomography (CT) imaging to predict remission following either a 6 or 12 week course of antibiotics for the non-surgical management of osteomyelitis of the foot in diabetes in a selected series of 45 individuals. They show that, in their series, if after 6 weeks of antibiotics the foot was free from signs of inflammation and the SPECT/CT scan was negative, there was no recurrence in the succeeding 12 months. If at 6 weeks there were either clinical signs of active disease or the scan was positive, antibiotics were continued for a further 6 weeks. The scan was then repeated at the end of the second course and the authors found that the result predicted later relapse in all six who were positive at that time-with a sensitivity, specificity, positive predictive value (PPV) and negative predictive value (NPV) of $100 \%, 56 \%, 46 \%$ and $100 \%$, respectively. In these circumstances, it can be concluded that a negative scan makes a relapse very unlikely. It is clearly important to be able to predict freedom from likely recurrence as soon as possible in order to reduce exposure to unnecessary antibiotic use. This work provides a useful basis on which to debate the limits of our current understanding of the issues relating to this common, and limb-threatening, complication of diabetes. 


\section{The diagnosis of osteomyelitis}

Osteomyelitis of the foot in diabetes is a clinical syndrome in which pathogenic bacteria gain access to bone by contiguous spread (almost always) before proliferating and causing local damage. The condition is much more common in the forefoot than the hindfoot (as in the paper by Vouillarmet and colleagues, where all but one case affected only the digits or metatarsals). Osteomyelitis is typically associated with clinical inflammation and swelling of the affected digit or part of the foot. Routine diagnosis relies on the association of this typical clinical picture with $\mathrm{x}$-ray evidence of underlying destruction of bone. It is well recognised, however, that plain $\mathrm{x}$-ray is relatively insensitive (especially in the early stages of the disease) and that MRI may be a more sensitive marker of marrow oedema (which may be helpful when larger bones are affected) and CT may be useful in highlighting fractures that are not apparent on plain x-ray. Even so, the diagnosis in the vast majority of cases worldwide will be based on clinical evidence of inflammation complicating a pre-existing ulcer and $\mathrm{x}$-ray confirmation.

The strength of the clinical signs was ranked some years ago by a working party of the International Working Group on the Diabetic Foot (IWGDF) according to the degree of support that they could lend to the diagnosis [2]. The direct observation of pus exuding from bone was sufficient evidence on its own, but most had to be considered in combination in order to derive a relative probability of bone infection. At the same time, the IWGDF proposed that the diagnosis should be substantiated by examination of a bone sample for both histological and microbiological changes, and this has since been regarded as the gold standard for the diagnosis of osteomyelitis [3], even though the necessary methodology and interpretation have never been formally described and their use for this purpose has not been evaluated. Bone biopsies were not routinely used in the Vouillarmet study and the diagnosis of osteomyelitis was based, as in everyday clinical practice, on clinical presentation supplemented by plain x-rays.

What about Charcot disease? The other important point to be made about the diagnosis of osteomyelitis relates to its differentiation from active Charcot disease - a circumstance that is rarely considered in papers describing the performance of different diagnostic tests. It is important because the two conditions are both characterised by inflammation and bone breakdown, and while the inflammatory changes of the Charcot foot become more chronic as the disease persists, both are typified by an acute phase inflammatory infiltration in early stages. Moreover, the two conditions may co-exist, either as a result of secondary infection of an ulcer complicating a Charcot deformity or, as is increasingly recognised in clinical practice, when the inflammation of infection appears to trigger the onset of Charcot foot disease. All clinicians need to have a low threshold for considering when either of these possibilities might apply (see Fig. 1).

\section{SPECT/CT scanning}

It is against this rather uncertain background that the emerging data on the diagnostic value of SPECT/CT in the diagnosis of osteomyelitis must be assessed. The technique essentially allows areas of inflammation on imaging to be linked to particular tissues with the customary anatomical precision of CT scanning. The source of photons may be an agent such as gallium-67 or may be attached in vitro to autologous white cells using, for example, technetium-99m before they are reinjected - as in the current study. In either case the photon source will obviously concentrate at areas of inflammation. The technique can produce images of great value but it is time consuming, expensive and not widely available.

SPECT/CT in the diagnosis of osteomyelitis One important recent publication used retrospective data collected from 166 people with diabetes complicated by osteomyelitis of the foot, and in whom biopsies had been undertaken and results were available for histology, microbiology or both [4]. Acknowledging that this was a selected population in which the overall disease prevalence was high at $67 \%$, the authors compared the diagnostic precision of ${ }^{99} \mathrm{Tc}$ WBC-SPECT/CT scanning with MRI in two parallel but comparable groups. They found that the sensitivity, specificity, PPV and NPV for both were virtually identical at $87 / 89 \%, 37 / 35 \%, 74 / 74 \%$ and
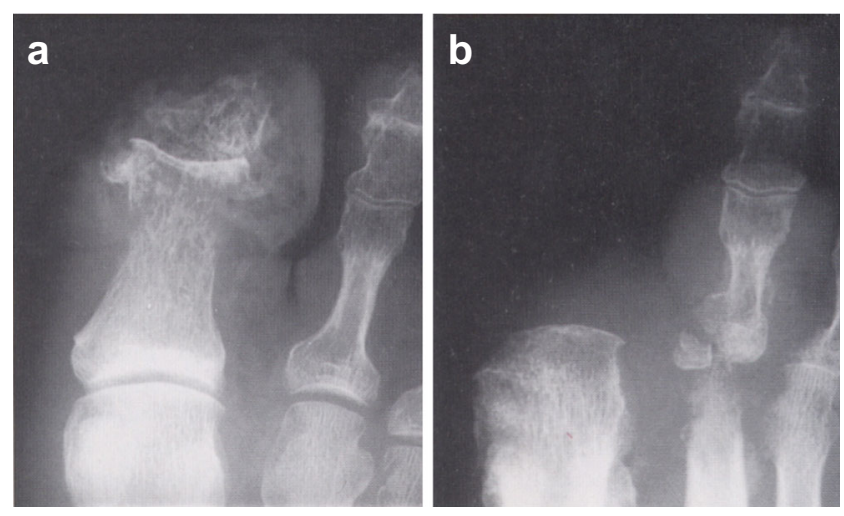

Fig. 1 This person underwent elective amputation of the hallux for osteomyelitis which was seemingly limited to the right hallux (a). The person returned after 6 months with recurrent inflammation of the forefoot, and the second x-ray (b) showed new changes in the head of the first metatarsal as well as in the second and third digits. While these changes would usually be attributed to osteomyelitis that had recurred because the extent of the original disease had not been recognised at the time of surgery, it is also possible that changes such as this could be the result of secondary Charcot disease 
$58 / 60 \%$. These data serve to support the diagnostic value of SPECT/CT but also indicate that it is little better than MRI for routine purposes; both have high sensitivity but limited specificity leading to appreciable numbers of false-positive results.

Another important study focused on the use of SPECT/CT scanning to diagnose osteomyelitis in an atypical group: those with chronic unhealing ulcers over bony prominences but with neither signs of clinical inflammation nor overt bone damage on X-ray [5]. This study used gallium-67 SPECT/CT to assess the possibility of osteomyelitis in a population of 55 people (all male) with non-healing foot ulcers. The scans were positive in 42 , and these underwent bone biopsy for microbiological analysis. Sixteen of the 42 were eventually judged to be culture-negative after examination of a number of repeat bone samples. Two were excluded, leaving 24 who were then treated with culture-guided antibiotics, and this led to cure or improvement in 19 (plus three amputations and two deaths). Those who were originally either scan-negative or biopsy-negative were left untreated and did not develop overt infection during 12 months of follow-up.

These data suggest that a negative scan and a negative bone culture are each associated with a very low risk of later occurrence of clinical osteomyelitis (i.e. each test has a high NPV) $[4,5]$, and the second paper also reveals that a negative bone culture is sufficient evidence on which to withhold antibiotics if there are no other clinical signs to suggest infection [5]. What they do not prove, however, is that the fairly good outcome in those who were scan- and microbiology-positive was the direct result of the use of antibiotics (because treatment was given to all of them). The only clear way to validate the assumption that either a positive SPECT/CT or a positive microbiological analysis of a biopsy is sufficient indication to use antibiotics in the absence of clinical signs of infection is to undertake a randomised trial in which half of those with positive scans/cultures receive no active treatment. This has not yet been done.

\section{SPECT/CT scanning in monitoring the response to} treatment of osteomyelitis In an earlier pilot study, Vouillarmet and colleagues compared the performance of plain x-ray, three-phase bone scan and WBC-SPECT/CT in predicting relapse in 29 people with osteomyelitis treated with systemic antibiotics [6]. All three approaches showed a high sensitivity and associated high NPV, but the WBC-SPECT/ CT had better specificity and PPV, at $91.5 \%$ and $71.5 \%$, respectively. A more recent study of 20 individuals by Lazaga et al [7] also reported the respectable predictive capacity of this technique, with sensitivity, specificity, PPV and NPV values of $90 \%, 65 \%, 69 \%$ and $83 \%$, respectively. And while it is unfortunate that none of these studies has calculated positive and negative likelihood ratios, which are less affected by pre-test probability than are both PPV and
NPV, it would appear from all of these reports that WBCSPECT/CT is both a reliable indicator of remission and relatively useful in the prediction of future short- to mediumterm relapse.

Does SPECT/CT imaging confer advantages over usual conventional monitoring? While there is some recent evidence to suggest that longitudinal monitoring of Creactive protein, erythrocyte sedimentation rate and other inflammatory markers may be of value in predicting clinical outcome $[8,9]$, the findings need to be confirmed and the methodology needs to be refined for prospective use. In the meantime, most clinicians pursue a pragmatic approach that relies to a large extent on the resolution of clinical signs of soft tissue inflammation. Game and Jeffcoate reported the use of such an approach in their retrospective review of outcome in 147 consecutive cases with osteomyelitis managed in routine clinical practice in a single centre [10]. A total of 34 of these 147 were judged to require early surgery, while systemic antibiotics (usually oral) were the mainstay of management of the remaining 113. It is interesting to note that in those selected in this way for management with antibiotics alone, the percentage that remained healed without surgery and using clinical observation as the only means of routine monitoring $(82 \%)$ was almost exactly the same as that reported here in association with the use of SPECT/CT (84\%). This triggers the question as to whether prediction of late relapse by scanning necessarily makes any difference to the long-term outcome, and it could be argued that the end result may be the same if the recurrence was simply treated when it became apparent. It is therefore possible that even if SPECT/CT were more widely available, the capacity for earlier prediction of continuing disease might not prove to have much impact in routine clinical practice.

\section{How is the optimal duration of antibiotic use to be determined for each individual?}

But the aim of the paper by Vouillarmet and colleagues was to emphasise the potential for SPECT/CT to lead to earlier recognition of remission and thus to reduced prescription of unnecessary antibiotics (with fewer adverse events and a lesser impact on the spread of antimicrobial resistance). While possible, this indirect benefit remains to be established and even if it is, the limited availability of SPECT/CT will remain a barrier - at least in the short term. More trials are urgently needed to extend the important finding of Tone and colleagues that a 6 week course of antibiotics was not inferior to a longer one [11] and, in particular, to determine whether antibiotic courses of just 2 or 3 weeks may be as effective in the management of osteomyelitis as they can be in the management of soft tissue infection. 
Duality of interest The author declares that there is no duality of interest associated with this manuscript.

Contribution statement The author is the sole contributor to this commentary.

\section{References}

1. Vouillarmet J, Moret M, Morelec I, Michon P, Dubreuil J (2017) Application of white blood cell SPECT/CT to predict remission after a 6 or 12 week course of antibiotic treatment for diabetic foot osteomyelitis. Diabetologia https://doi.org/10.1007/s00125017-4417-x

2. Berendt AR, Peters EJ, Bakker K et al (2008) Diabetic foot osteomyelitis: a progress report on diagnosis and a systematic review of treatment. Diabetes Metab Res Rev 24(Supp11):S145-S161

3. Lipsky BA, Aragon-Sanchez J, Diggle M et al (2016) IWGDF guidance on the diagnosis and management of foot infections in persons with diabetes. Diabetes Metab Res Rev 32(Suppl1):45-74

4. La Fontaine J, Bhavan K, Lam K et al (2016) Comparison between Tc-99m WBC SPECT/CT and MRI for the diagnosis of biopsyproven diabetic foot osteomyelitis. Wounds 28:271-278
5. Aslangul E, M'bemba J, Caillat-Vigneron N et al (2013) Diagnosing soft tissue osteomyelitis in patients without signs of soft tissue infection by coupling hybrid ${ }^{67} \mathrm{Ga}$ SPECT/CT with bedside percutaneous bone puncture. Diabetes Care 36:2203-2210

6. Vouillarmet J, Morelec I, Thivolet C (2014) Assessing diabetic foot osteomyelitis remission with white blood cell SPECT/CT imaging. Diabet Med 31:1093-1099

7. Lazaga F, Van Asten SA, Nichols A et al (2016) Hybrid imaging with $99 \mathrm{mTc}-W B C$ SPECT/CT to monitor the effect of therapy in diabetic foot osteomyelitis. Int Wound J 13:1158-1160

8. van Asten SA, Jupiter DC, Mithani M, La Fontaine J, David KE, Lavery LA (2017) Erythrocyte sedimentation rate and C-reactive protein to monitor treatment outcomes in diabetic foot osteomyelitis. Int Wound J 14:142-148

9. Van Asten SA, Nichols A, La Fontaine J, Bhavan K, Peters EJ, Lavery LA (2017) The value of inflammatory markers to diagnose and monitor diabetic foot osteomyelitis. Int Wound J 14:40-45

10. Game FL, Jeffcoate WJ (2008) Primarily non-surgical management of osteomyelitis of the foot in diabetes. Diabetologia 51:962-967

11. Tone A, Nguyen S, Devemy F et al (2015) Six-week versus twelve week antibiotic therapy for nonsurgically treated diabetic foot osteomyelitis: a multicenter open-label controlled randomized study. Diabetes Care 38:302-307 